\title{
Green's function for Sturm-Liouville-type boundary value problems of fractional order impulsive differential equations and its application
}

Jie Zhou and Meiqiang Feng*

*Correspondence:

meiqiangfeng@sina.com School of Applied Science, Beijing Information Science \& Technology University, Beijing, 100192, Republic of China

\begin{abstract}
In this paper, we discuss the expression and properties of Green's function for boundary value problems of nonlinear Sturm-Liouville-type fractional order impulsive differential equations. Its applications are also given. Our results are compared with some recent results by Bai and Lü.
\end{abstract}

Keywords: fractional differential equation; impulse; Green's function; existence; fixed point theorem

\section{Introduction}

Fractional differential equations arise in many engineering and scientific disciplines as the mathematical modeling of systems and processes in the fields of physics, chemistry, aerodynamics, electrodynamics of complex medium, polymer rheology, Bode's analysis of feedback amplifiers, capacitor theory, electrical circuits, electron-analytical chemistry, biology, control theory, fitting of experimental data, and so forth, and involves derivatives of fractional order. Fractional derivatives provide an excellent tool for the description of memory and hereditary properties of various materials and processes. This is the main advantage of fractional differential equations in comparison with classical integer-order models. An excellent account in the study of fractional differential equations can be found in [1-5]. For the basic theory and recent development of the subject, we refer to a text by Lakshmikantham et al. [6]. For more details and examples, see [7-23] and the references therein.

Integer-order impulsive differential equations have become important in recent years as mathematical models of phenomena in both physical and social sciences. There has been a significant development in impulsive theory especially in the area of impulsive differential equations with fixed moments; see, for instance, [24-26]. Recently, the boundary value problems of impulsive differential equations of integer order have been studied extensively in the literature (see [27-36]).

On the other hand, impulsive differential equations of fractional order play an important role in theory and applications, see [37-46] and the references therein. However, as pointed out in $[38,39]$, the fractional impulsive differential equations have not been ad-

○2014 Zhou and Feng; licensee Springer. This is an Open Access article distributed under the terms of the Creative Commons Attribution License (http://creativecommons.org/licenses/by/2.0), which permits unrestricted use, distribution, and reproduction in any medium, provided the original work is properly cited. 
dressed so extensively and many aspects of these problems are yet to be explored. For example, the theory using Green's function to express the solution of fractional impulsive differential equations has not been investigated till now. Now, in this paper, we shall study the expression of the solution of fractional impulsive differential equations by using Green's function.

Consider the following nonlinear boundary value problem of fractional impulsive differential equations:

$$
\left\{\begin{array}{l}
{ }^{c} \mathbf{D}_{0+}^{q} x(t)=\omega(t) f\left(t, x(t), x^{\prime}(t)\right), \quad 1<q \leq 2, t \in J_{1}=J \backslash\left\{t_{1}, t_{2}, \ldots, t_{n}\right\}, \\
\left.\Delta x\right|_{t=t_{k}}=\mathcal{I}_{k}\left(x\left(t_{k}\right)\right),\left.\quad \Delta x^{\prime}\right|_{t=t_{k}}=\mathcal{J}_{k}\left(x\left(t_{k}\right)\right), \quad t_{k} \in(0,1), k=1,2, \ldots, n, \\
\alpha_{1} x(0)-\beta_{1} x^{\prime}(0)=0, \quad \alpha_{2} x(1)+\beta_{2} x^{\prime}(1)=0,
\end{array}\right.
$$

where ${ }^{c} \mathbf{D}_{0+}^{q}$ is the Caputo fractional derivative, $J=[0,1], \omega(t): J \rightarrow R+$ is a continuous function, $f: J \times R \times R \rightarrow R$ is a continuous function, $\mathcal{I}_{k}, \mathcal{J}_{k}: R \rightarrow R$ are continuous functions, $\alpha_{1}, \alpha_{2}, \beta_{1}, \beta_{2} \in R$ and $\alpha_{1} \alpha_{2}+\alpha_{1} \beta_{2}+\alpha_{2} \beta_{1} \neq 0 .\left.\Delta x\right|_{t=t_{k}}=x\left(t_{k}^{+}\right)-x\left(t_{k}^{-}\right)$with $x\left(t_{k}^{+}\right)=$ $\lim _{h \rightarrow 0^{+}} x\left(t_{k}+h\right), x\left(t_{k}^{-}\right)=\lim _{h \rightarrow 0^{-}} x\left(t_{k}+h\right), k=1,2, \ldots, n, 0=t_{0}<t_{1}<\cdots<t_{n}<t_{n+1}=1$. $\left.\Delta x^{\prime}\right|_{t=t_{k}}$ has a similar meaning for $x^{\prime}(t)$.

Some special cases of (1.1) have been investigated. For example, Bai and Lü [13] considered problem (1.1) with $\mathcal{I}_{k} \equiv 0$ and $\mathcal{J}_{k} \equiv 0$. By using the fixed point theorem in cones, they proved some existence and multiplicity results of positive solutions of problem (1.1).

At the end of this section, it is worth mentioning that it is an important method to express the solution of differential equations by Green's function. According to the previous work, we find that the solution of impulsive differential equations with integer order can be expressed by Green's function of the case without impulse. For example, Green's function of the following boundary value problem

$$
\left\{\begin{array}{l}
-x^{\prime \prime}(t)=\sigma(t), \quad t \in(0,1) \\
x(0)=x(1)=0
\end{array}\right.
$$

can be expressed by

$$
G(t, s)= \begin{cases}s(1-t), & 0 \leq s \leq t \leq 1 \\ t(1-s), & 0 \leq t \leq s \leq 1\end{cases}
$$

where $\sigma \in C[0,1]$. The solution of problem (1.2) can be expressed by

$$
x(t)=\int_{0}^{1} G(t, s) \sigma(s) d s .
$$

If we consider impulsive differential equations

$$
\left\{\begin{array}{l}
-x^{\prime \prime}(t)=\sigma(t), \quad t \in(0,1), t \neq t_{k}, \\
\left.\Delta x\right|_{t=t_{k}}=\mathcal{I}_{k}\left(x\left(t_{k}\right)\right), \quad k=1,2, \ldots, n, \\
\left.\Delta x^{\prime}\right|_{t=t_{k}}=-\mathcal{L}_{k}\left(x\left(t_{k}\right)\right), \quad k=1,2, \ldots, n, \\
x(0)=x(1)=0,
\end{array}\right.
$$


then the solution of problem (1.3) can be expressed by

$$
x(t)=\int_{0}^{1} G(t, s) \sigma(s) d s+\sum_{k=1}^{n} G\left(t, t_{k}\right) \mathcal{L}_{k}\left(x_{t_{k}}\right)+\sum_{k=1}^{n} G_{s}^{\prime}\left(t, t_{k}\right) \mathcal{I}_{k}\left(x_{t_{k}}\right),
$$

where $\mathcal{I}_{k}, \mathcal{L}_{k} \in C[0,1], k=1,2, \ldots, n$.

Naturally, one wishes to know whether or not the same result holds for the fractional order case. We first study the fractional order differential equations with Caputo derivatives

$$
\left\{\begin{array}{l}
-{ }^{c} \mathbf{D}_{0+}^{q} x(t)=\sigma(t), \quad t \in(0,1), 1<q \leq 2, \\
x(0)=x(1)=0,
\end{array}\right.
$$

where ${ }^{c} \mathbf{D}_{0+}^{q}$ is the Caputo fractional derivative. Then

$$
x(t)=\int_{0}^{1} G(t, s) \sigma(s) d s,
$$

where

$$
G(t, s)=\frac{1}{\Gamma(q)} \begin{cases}t(1-s)^{q-1}-(t-s)^{q-1}, & 0 \leq s \leq t \leq 1 \\ t(1-s)^{q-1}, & 0 \leq t \leq s \leq 1 .\end{cases}
$$

Then we study whether the solution of fractional order impulsive differential equations with Caputo derivatives

$$
\left\{\begin{array}{l}
-{ }^{c} \mathbf{D}_{0+}^{q} x(t)=\sigma(t), \quad t \in(0,1), 1<q \leq 2, \\
\left.\Delta x\right|_{t=t_{k}}=\mathcal{I}_{k}\left(x\left(t_{k}\right)\right), \quad k=1,2, \ldots, n, \\
\left.\Delta x^{\prime}\right|_{t=t_{k}}=-\mathcal{L}_{k}\left(x\left(t_{k}\right)\right), \quad k=1,2, \ldots, n, \\
x(0)=x(1)=0
\end{array}\right.
$$

can be expressed by

$$
x(t)=\int_{0}^{1} G(t, s) \sigma(s) d s+\sum_{k=1}^{n} G\left(t, t_{k}\right) \mathcal{L}_{k}\left(x_{t_{k}}\right)+\sum_{k=1}^{n} G_{s}^{\prime}\left(t, t_{k}\right) \mathcal{I}_{k}\left(x_{t_{k}}\right) .
$$

Thus, it is an interesting problem, and so it is worthwhile to study. We will give the answers in the following sections.

The organization of this paper is as follows. In Section 2, we present the expression and properties of Green's function associated with problem (1.1). In Section 3, we give some preliminaries about the operator and the fixed point theorem. In Section 4, we get some existence results for problem (1.1) by means of some standard fixed point theorems. The final section of the paper contains two examples to illustrate our main results. 


\section{Expression and properties of Green's function}

Consider the following fractional impulsive boundary value problem:

$$
\left\{\begin{array}{l}
{ }^{c} \mathbf{D}_{0+}^{q} x(t)=\sigma(t), \quad 1<q \leq 2, t \in J_{1}=J \backslash\left\{t_{1}, t_{2}, \ldots, t_{n}\right\}, \\
\left.\Delta x\right|_{t=t_{k}}=\mathcal{I}_{k}\left(x\left(t_{k}\right)\right),\left.\quad \Delta x^{\prime}\right|_{t=t_{k}}=\mathcal{J}_{k}\left(x\left(t_{k}\right)\right), \quad t_{k} \in(0,1), k=1,2, \ldots, n, \\
\alpha_{1} x(0)-\beta_{1} x^{\prime}(0)=0, \quad \alpha_{2} x(1)+\beta_{2} x^{\prime}(1)=0,
\end{array}\right.
$$

where ${ }^{c} \mathbf{D}_{0+}^{q}$ is the Caputo fractional derivative, $\sigma(t) \in C[0,1], \mathcal{I}_{k}, \mathcal{J}_{k}: R \rightarrow R$ are continuous functions, $\alpha_{1}, \alpha_{2}, \beta_{1}, \beta_{2} \in R$ and $\alpha_{1} \alpha_{2}+\alpha_{1} \beta_{2}+\alpha_{2} \beta_{1} \neq 0 .\left.\Delta x\right|_{t=t_{k}}=x\left(t_{k}^{+}\right)-x\left(t_{k}^{-}\right)$ with $x\left(t_{k}^{+}\right)=\lim _{h \rightarrow 0^{+}} x\left(t_{k}+h\right), x\left(t_{k}^{-}\right)=\lim _{h \rightarrow 0^{-}} x\left(t_{k}+h\right), k=1,2, \ldots, n, 0=t_{0}<t_{1}<\cdots<$ $t_{n}<t_{n+1}=1 .\left.\Delta x^{\prime}\right|_{t=t_{k}}$ has a similar meaning for $x^{\prime}(t)$.

Theorem 2.1 The solution of problem (2.1) can be expressed by

$$
\begin{aligned}
x(t)= & \int_{t_{k}}^{t} \frac{(t-s)^{q-1}}{\Gamma(q)} \sigma(s) d s+\sum_{i=1}^{n+1} G_{1 s}^{\prime}\left(t, t_{i}\right) \int_{t_{i-1}}^{t_{i}} \frac{\left(t_{i}-s\right)^{q-1}}{\Gamma(q)} \sigma(s) d s \\
& -\sum_{i=1}^{n} G_{1}\left(t, t_{i}\right) \int_{t_{i-1}}^{t_{i}} \frac{\left(t_{i}-s\right)^{q-2}}{\Gamma(q-1)} \sigma(s) d s+\sum_{i=1}^{n} G_{1 s}^{\prime}\left(t, t_{i}\right) \mathcal{I}_{i}\left(x\left(t_{i}\right)\right) \\
& -\sum_{i=1}^{n} G_{1}\left(t, t_{i}\right) \mathcal{J}_{i}\left(x\left(t_{i}\right)\right), \quad t \in\left(t_{k}, t_{k+1}\right], k=0,1,2, \ldots, n, t_{0}=0, t_{n+1}=1,
\end{aligned}
$$

where

$$
G_{1}(t, s)=-\frac{1}{\eta} \begin{cases}\left(\beta_{1}+\alpha_{1} t\right)\left(\alpha_{2}+\beta_{2}-\alpha_{2} s\right), & t \leq s \\ \left(\beta_{1}+\alpha_{1} s\right)\left(\alpha_{2}+\beta_{2}-\alpha_{2} t\right), & t \geq s\end{cases}
$$

and

$$
\eta=\alpha_{1} \alpha_{2}+\alpha_{1} \beta_{2}+\alpha_{2} \beta_{1}
$$

Proof Suppose that $x$ is a solution of (2.1). Then, for some constants $b_{0}, b_{1} \in R$, we have

$$
x(t)=I_{0^{+}}^{q} \sigma(t)-b_{0}-b_{1} t=\int_{0}^{t} \frac{(t-s)^{q-1}}{\Gamma(q)} \sigma(s) d s-b_{0}-b_{1} t, \quad t \in\left[0, t_{1}\right] .
$$

It follows from (2.5) that

$$
x^{\prime}(t)=\int_{0}^{t} \frac{(t-s)^{q-2}}{\Gamma(q-1)} \sigma(s) d s-b_{1} .
$$

If $t \in\left(t_{1}, t_{2}\right]$, then, for some constants $c_{0}, c_{1} \in R$, we can write

$$
\begin{aligned}
& x(t)=\int_{t_{1}}^{t} \frac{(t-s)^{q-1}}{\Gamma(q)} \sigma(s) d s-c_{0}-c_{1}\left(t-t_{1}\right), \\
& x^{\prime}(t)=\int_{t_{1}}^{t} \frac{(t-s)^{q-2}}{\Gamma(q-1)} \sigma(s) d s-c_{1} .
\end{aligned}
$$


Using the impulse conditions $\left.\Delta x\right|_{t=t_{1}}=\mathcal{I}_{1}\left(x\left(t_{1}\right)\right)$ and $\left.\Delta x^{\prime}\right|_{t=t_{1}}=\mathcal{J}_{1}\left(x\left(t_{1}\right)\right)$, we find that

$$
\begin{aligned}
& -c_{0}=\int_{0}^{t_{1}} \frac{\left(t_{1}-s\right)^{q-1}}{\Gamma(q)} \sigma(s) d s-b_{0}-b_{1} t_{1}+\mathcal{I}_{1}\left(x\left(t_{1}\right)\right), \\
& -c_{1}=\int_{0}^{t_{1}} \frac{\left(t_{1}-s\right)^{q-2}}{\Gamma(q-1)} \sigma(s) d s-b_{1}+\mathcal{J}_{1}\left(x\left(t_{1}\right)\right) .
\end{aligned}
$$

Thus

$$
\begin{aligned}
x(t)= & \int_{t_{1}}^{t} \frac{(t-s)^{q-1}}{\Gamma(q)} \sigma(s) d s+\int_{0}^{t_{1}}\left(\frac{\left(t-t_{1}\right)\left(t_{1}-s\right)^{q-2}}{\Gamma(q-1)}+\frac{\left(t_{1}-s\right)^{q-1}}{\Gamma(q)}\right) \sigma(s) d s \\
& -b_{0}-b_{1} t+\mathcal{J}_{1}\left(x\left(t_{1}\right)\right)\left(t-t_{1}\right)+\mathcal{I}_{1}\left(x\left(t_{1}\right)\right), \\
x^{\prime}(t)= & \int_{t_{1}}^{t} \frac{(t-s)^{q-2}}{\Gamma(q-1)} \sigma(s) d s+\int_{0}^{t_{1}} \frac{\left(t_{1}-s\right)^{q-2}}{\Gamma(q-1)} \sigma(s) d s-b_{1}+\mathcal{J}_{1}\left(x\left(t_{1}\right)\right) .
\end{aligned}
$$

If $t \in\left(t_{k}, t_{k+1}\right]$, repeating the above procedure, we obtain

$$
\begin{aligned}
x(t)= & \int_{t_{k}}^{t} \frac{(t-s)^{q-1}}{\Gamma(q)} \sigma(s) d s+\sum_{i=1}^{k} \int_{t_{i-1}}^{t_{i}}\left(\frac{\left(t-t_{i}\right)\left(t_{i}-s\right)^{q-2}}{\Gamma(q-1)}+\frac{\left(t_{i}-s\right)^{q-1}}{\Gamma(q)}\right) \sigma(s) d s \\
& -b_{0}-b_{1} t+\sum_{i=1}^{k} \mathcal{J}_{i}\left(x\left(t_{i}\right)\right)\left(t-t_{i}\right)+\sum_{i=1}^{k} \mathcal{I}_{i}\left(x\left(t_{i}\right)\right) .
\end{aligned}
$$

It follows that $x(0)=-b_{0}, x^{\prime}(0)=-b_{1}$ and

$$
\begin{aligned}
x(1)= & \int_{t_{n}}^{1} \frac{(1-s)^{q-1}}{\Gamma(q)} \sigma(s) d s+\sum_{i=1}^{n} \int_{t_{i-1}}^{t_{i}}\left(\frac{\left(1-t_{i}\right)\left(t_{i}-s\right)^{q-2}}{\Gamma(q-1)}+\frac{\left(t_{i}-s\right)^{q-1}}{\Gamma(q)}\right) \sigma(s) d s \\
& -b_{0}-b_{1}+\sum_{i=1}^{n} \mathcal{J}_{i}\left(x\left(t_{i}\right)\right)\left(1-t_{i}\right)+\sum_{i=1}^{n} \mathcal{I}_{i}\left(x\left(t_{i}\right)\right), \\
x^{\prime}(1)= & \int_{t_{n}}^{1} \frac{(1-s)^{q-2}}{\Gamma(q-1)} \sigma(s) d s+\sum_{i=1}^{n} \int_{t_{i-1}}^{t_{i}} \frac{\left(t_{i}-s\right)^{q-2}}{\Gamma(q-1)} \sigma(s) d s-b_{1}+\sum_{i=1}^{n} \mathcal{J}_{i}\left(x\left(t_{i}\right)\right) .
\end{aligned}
$$

By the boundary conditions, we have

$$
\begin{aligned}
b_{0}= & \frac{1}{\eta}\left\{\alpha _ { 2 } \beta _ { 1 } \left[\int_{t_{n}}^{1} \frac{(1-s)^{q-1}}{\Gamma(q)} \sigma(s) d s\right.\right. \\
& +\sum_{i=1}^{n} \int_{t_{i-1}}^{t_{i}}\left(\frac{\left(1-t_{i}\right)\left(t_{i}-s\right)^{q-2}}{\Gamma(q-1)}+\frac{\left(t_{i}-s\right)^{q-1}}{\Gamma(q)}\right) \sigma(s) d s \\
& \left.+\sum_{i=1}^{n} \mathcal{J}_{i}\left(x\left(t_{i}\right)\right)\left(1-t_{i}\right)+\sum_{i=1}^{n} \mathcal{I}_{i}\left(x\left(t_{i}\right)\right)\right]+\beta_{1} \beta_{2}\left[\int_{t_{n}}^{1} \frac{(1-s)^{q-2}}{\Gamma(q-1)} \sigma(s) d s\right. \\
& \left.\left.+\sum_{i=1}^{n} \int_{t_{i-1}}^{t_{i}} \frac{\left(t_{i}-s\right)^{q-2}}{\Gamma(q-1)} \sigma(s) d s+\sum_{i=1}^{n} \mathcal{J}_{i}\left(x\left(t_{i}\right)\right)\right]\right\} \\
b_{1}= & \frac{1}{\eta}\left\{\alpha _ { 1 } \alpha _ { 2 } \left[\int_{t_{n}}^{1} \frac{(1-s)^{q-1}}{\Gamma(q)} \sigma(s) d s+\sum_{i=1}^{n} \int_{t_{i-1}}^{t_{i}}\left(\frac{\left(1-t_{i}\right)\left(t_{i}-s\right)^{q-2}}{\Gamma(q-1)}+\frac{\left(t_{i}-s\right)^{q-1}}{\Gamma(q)}\right) \sigma(s) d s\right.\right.
\end{aligned}
$$




$$
\begin{aligned}
& \left.+\sum_{i=1}^{n} \mathcal{J}_{i}\left(x\left(t_{i}\right)\right)\left(1-t_{i}\right)+\sum_{i=1}^{n} \mathcal{I}_{i}\left(x\left(t_{i}\right)\right)\right]+\alpha_{1} \beta_{2}\left[\int_{t_{n}}^{1} \frac{(1-s)^{q-2}}{\Gamma(q-1)} \sigma(s) d s\right. \\
& \left.\left.+\sum_{i=1}^{n} \int_{t_{i-1}}^{t_{i}} \frac{\left(t_{i}-s\right)^{q-2}}{\Gamma(q-1)} \sigma(s) d s+\sum_{i=1}^{n} \mathcal{J}_{i}\left(x\left(t_{i}\right)\right)\right]\right\},
\end{aligned}
$$

where $\eta$ is defined in (2.4).

Substituting (2.7), (2.8) into (2.6), we obtain (2.2). This completes the proof.

Remark 2.1 It is clear that $G_{1}(t, s)$ is Green's function of the boundary value problem

$$
\left\{\begin{array}{l}
x^{\prime \prime}(t)=\sigma(t), \quad t \in(0,1) \\
\alpha_{1} x(0)-\beta_{1} x^{\prime}(0)=0 \\
\alpha_{2} x(1)+\beta_{2} x^{\prime}(1)=0
\end{array}\right.
$$

Remark 2.2 The expression of the solution of problem (2.1) is simpler than that of [3746].

From (2.3), we can prove the following results.

Proposition 2.1 For all $t, s \in J$, we have

$$
\left|G_{1}(t, s)\right| \leq \frac{2\left(\left|\beta_{1} \alpha_{2}\right|+\left|\alpha_{1} \alpha_{2}\right|\right)+\left|\beta_{1} \beta_{2}\right|+\left|\alpha_{1} \beta_{2}\right|}{|\eta|}
$$

Proposition 2.2 For all $t, s \in J$, we have

$$
\begin{aligned}
& \left|G_{1 s}^{\prime}(t, s)\right| \leq \max \left\{\frac{\left|\alpha_{2} \beta_{1}\right|+\left|\alpha_{1} \alpha_{2}\right|}{|\eta|}, \frac{\left|\alpha_{1} \beta_{2}\right|+2\left|\alpha_{1} \alpha_{2}\right|}{|\eta|}\right\}, \\
& \left|G_{1 t}^{\prime}(t, s)\right| \leq \max \left\{\frac{\left|\alpha_{1} \beta_{2}\right|+2\left|\alpha_{1} \alpha_{2}\right|}{|\eta|}, \frac{\left|\alpha_{2} \beta_{1}\right|+\left|\alpha_{1} \alpha_{2}\right|}{|\eta|}\right\}
\end{aligned}
$$

and

$$
\left|G_{1 s t}^{\prime \prime}(t, s)\right|=\frac{\left|\alpha_{1} \alpha_{2}\right|}{|\eta|}
$$

where

$$
\begin{aligned}
& G_{1 s}^{\prime}(t, s)=-\frac{1}{\eta} \begin{cases}-\alpha_{2}\left(\beta_{1}+\alpha_{1} t\right), & t \leq s, \\
\alpha_{1}\left(\alpha_{2}+\beta_{2}-\alpha_{2} t\right), & t \geq s,\end{cases} \\
& G_{1 t}^{\prime}(t, s)=-\frac{1}{\eta} \begin{cases}\alpha_{1}\left(\alpha_{2}+\beta_{2}-\alpha_{2} s\right), & t \leq s, \\
-\alpha_{2}\left(\beta_{1}+\alpha_{1} s\right), & t \geq s,\end{cases}
\end{aligned}
$$

and

$$
G_{1 s t}^{\prime \prime}(t, s)=\frac{\alpha_{1} \alpha_{2}}{\eta}
$$


For the sake of convenience, let

$$
\begin{aligned}
& c_{1}=\frac{2\left(\left|\beta_{1} \alpha_{2}\right|+\left|\alpha_{1} \alpha_{2}\right|\right)+\left|\beta_{1} \beta_{2}\right|+\left|\alpha_{1} \beta_{2}\right|}{|\eta|}, \\
& c_{2}=\max \left\{\frac{\left|\alpha_{2} \beta_{1}\right|+\left|\alpha_{1} \alpha_{2}\right|}{|\eta|}, \frac{\left|\alpha_{1} \beta_{2}\right|+2\left|\alpha_{1} \alpha_{2}\right|}{|\eta|}\right\}, \quad c_{3}=\frac{\left|\alpha_{1} \alpha_{2}\right|}{|\eta|} .
\end{aligned}
$$

Then it follows from (2.9) and (2.10) that

$$
\left|G_{1}(t, s)\right| \leq c_{1}, \quad\left|G_{1 t}^{\prime}(t, s)\right| \leq c_{2}, \quad\left|G_{1 s}^{\prime}(t, s)\right| \leq c_{2}, \quad\left|G_{1 s t}^{\prime}(t, s)\right| \leq c_{3} .
$$

From the proof of Theorem 2.1 we have the following results.

Proposition 2.3 The solution of fractional impulsive differential equations can be expressed by Green's function, and it is not Green's function of the corresponding fractional differential equations, but Green's function of the corresponding integer order differential equations.

\section{Preliminaries}

In this section, we give some preliminaries for discussing the solvability of problem (1.1) as follows.

$$
\begin{aligned}
& \text { Let } \begin{aligned}
& J^{\prime}=[0,1] \backslash\left\{t_{1}, t_{2}, \ldots, t_{n}\right\} \text { and } \\
& P C[, R]=\left\{x: J \rightarrow R ; x \in C\left(\left(t_{k}, t_{k+1}\right), R\right), x\left(t_{k}^{+}\right) \text {and } x\left(t_{k}^{-}\right) \text {exist with } x\left(t_{k}^{-}\right)=x\left(t_{k}\right),\right. \\
&k=1,2, \ldots, n\}, \\
& P C^{1}[J, R]=\left\{x^{\prime} \in P C J, R\right] ; x^{\prime}\left(t_{k}^{+}\right), x\left(t_{k}^{-}\right) \text {exist and } x^{\prime} \text { is left continuous at } t_{k}, \\
&k=1,2, \ldots, n\} .
\end{aligned}
\end{aligned}
$$

Then $P C[J, R]$ is a Banach space with the norm

$$
\|x\|_{P C}=\sup _{t \in J}|x(t)|
$$

$P C^{1}[J, R]$ is a Banach space with the norm

$$
\|x\|_{P C^{1}}=\max \left\{\|x\|_{P C},\left\|x^{\prime}\right\|_{P C}\right\}
$$

Definition 3.1 A function $x \in P C^{1}[J, R] \cap C^{2}\left[J^{\prime}, R\right]$ with its Caputo derivative of order $q$ existing on $J$ is a solution of problem (1.1) if it satisfies (1.1).

We give the following hypotheses:

$\left(\mathrm{H}_{1}\right) \omega: J \rightarrow[0,+\infty)$ is a continuous function, and there exists $t_{0} \in J$ such that $\omega\left(t_{0}\right)>0$;

$\left(\mathrm{H}_{2}\right) f: J \times R \times R \rightarrow R$ is a continuous function;

$\left(\mathrm{H}_{3}\right) \mathcal{I}_{k}, \mathcal{J}_{k}: R \rightarrow R$ are continuous functions.

It follows from Theorem 2.1 that: 
Lemma 3.1 If $\left(\mathrm{H}_{1}\right)-\left(\mathrm{H}_{3}\right)$ hold, then a function $x \in P C^{1}[J, R] \cap C^{2}\left[J^{\prime}, R\right]$ is a solution of problem (1.1) if and only if $x \in P C^{1}[U, R]$ is a solution of the impulsive fractional integral equation

$$
\begin{aligned}
x(t)= & \int_{t_{k}}^{t} \frac{(t-s)^{q-1}}{\Gamma(q)} \omega(s) f\left(s, x(s), x^{\prime}(s)\right) d s \\
& +\sum_{i=1}^{n+1} G_{1 s}^{\prime}\left(t, t_{i}\right) \int_{t_{i-1}}^{t_{i}} \frac{\left(t_{i}-s\right)^{q-1}}{\Gamma(q)} \omega(s) f\left(s, x(s), x^{\prime}(s)\right) d s \\
& -\sum_{i=1}^{n} G_{1}\left(t, t_{i}\right) \int_{t_{i-1}}^{t_{i}} \frac{\left(t_{i}-s\right)^{q-2}}{\Gamma(q-1)} \omega(s) f\left(s, x(s), x^{\prime}(s)\right) d s \\
& +\sum_{i=1}^{n} G_{1 s}^{\prime}\left(t, t_{i}\right) \mathcal{I}_{i}\left(x\left(t_{i}\right)\right)-\sum_{i=1}^{n} G_{1}\left(t, t_{i}\right) \mathcal{J}_{i}\left(x\left(t_{i}\right)\right), \\
& t \in\left(t_{k}, t_{k+1}\right], k=0,1,2, \ldots, n, t_{0}=0, t_{n+1}=1 .
\end{aligned}
$$

Define $T: P C^{1}[J, R] \rightarrow P C^{1}[J, R]$ by

$$
\begin{aligned}
(T x)(t)= & \int_{t_{k}}^{t} \frac{(t-s)^{q-1}}{\Gamma(q)} \omega(s) f\left(s, x(s), x^{\prime}(s)\right) d s \\
& +\sum_{i=1}^{n+1} G_{1 s}^{\prime}\left(t, t_{i}\right) \int_{t_{i-1}}^{t_{i}} \frac{\left(t_{i}-s\right)^{q-1}}{\Gamma(q)} \omega(s) f\left(s, x(s), x^{\prime}(s)\right) d s \\
& -\sum_{i=1}^{n} G_{1}\left(t, t_{i}\right) \int_{t_{i-1}}^{t_{i}} \frac{\left(t_{i}-s\right)^{q-2}}{\Gamma(q-1)} \omega(s) f\left(s, x(s), x^{\prime}(s)\right) d s \\
& +\sum_{i=1}^{n} G_{1 s}^{\prime}\left(t, t_{i}\right) \mathcal{I}_{i}\left(x\left(t_{i}\right)\right)-\sum_{i=1}^{n} G_{1}\left(t, t_{i}\right) \mathcal{J}_{i}\left(x\left(t_{i}\right)\right), \\
& t \in\left(t_{k}, t_{k+1}\right], k=0,1,2, \ldots, n, t_{0}=0, t_{n+1}=1 .
\end{aligned}
$$

Using Lemma 3.1, problem (1.1) reduces to a fixed point problem $x=T x$, where $T$ is given by (3.2). Thus problem (1.1) has a solution if and only if the operator $T$ has a fixed point.

From (3.2) and Lemma 3.1, it is easy to obtain the following result.

Lemma 3.2 Assume that $\left(\mathrm{H}_{1}\right)-\left(\mathrm{H}_{3}\right)$ hold. Then $T: P C^{1}[J, R] \rightarrow P C^{1}[J, R]$ is completely continuous.

Proof Note that the continuity of $f, \omega, \mathcal{I}_{k}$ and $\mathcal{J}_{k}$ together with $G_{1}(t, s)$ and $G_{1 s}^{\prime}(t, s)$ ensures the continuity of $T$.

Let $\Omega \subset P C^{1}[, R]$ be bounded. Then there exist positive constants $\mu_{1}, \mu_{2}$ and $\mu_{3}$ such that $\left|f\left(t, x(t), x^{\prime}(t)\right)\right| \leq \mu_{1},\left|\mathcal{I}_{k}(x)\right| \leq \mu_{2}$ and $\left|\mathcal{J}_{k}(x)\right| \leq \mu_{3}, \forall x \in \Omega$. Thus, $\forall x \in \Omega$, we have

$$
\begin{aligned}
|(T x)(t)| \leq & \int_{t_{k}}^{t} \frac{(t-s)^{q-1}}{\Gamma(q)} \omega(s)\left|f\left(s, x(s), x^{\prime}(s)\right)\right| d s \\
& +\sum_{i=1}^{n+1}\left|G_{1 s}^{\prime}\left(t, t_{i}\right)\right| \int_{t_{i-1}}^{t_{i}} \frac{\left(t_{i}-s\right)^{q-1}}{\Gamma(q)} \omega(s)\left|f\left(s, x(s), x^{\prime}(s)\right)\right| d s
\end{aligned}
$$




$$
\begin{aligned}
& +\sum_{i=1}^{n}\left|G_{1}\left(t, t_{i}\right)\right| \int_{t_{i-1}}^{t_{i}} \frac{\left(t_{i}-s\right)^{q-2}}{\Gamma(q-1)} \omega(s)\left|f\left(s, x(s), x^{\prime}(s)\right)\right| d s \\
& +\sum_{i=1}^{n}\left|G_{1 s}^{\prime}\left(t, t_{i}\right)\right|\left|\mathcal{I}_{i}\left(x\left(t_{i}\right)\right)\right|+\sum_{i=1}^{n}\left|G_{1}\left(t, t_{i}\right)\right|\left|\mathcal{J}_{i}\left(x\left(t_{i}\right)\right)\right| \\
& \leq \frac{\gamma \mu_{1}}{\Gamma(q)} \int_{t_{k}}^{t}(t-s)^{q-1} d s+\frac{\gamma \mu_{1}}{\Gamma(q)} \sum_{i=1}^{n+1}\left|G_{1 s}^{\prime}\left(t, t_{i}\right)\right| \int_{t_{i-1}}^{t_{i}}\left(t_{i}-s\right)^{q-1} d s \\
& +\frac{\gamma \mu_{1}}{\Gamma(q-1)} \sum_{i=1}^{n}\left|G_{1}\left(t, t_{i}\right)\right| \int_{t_{i-1}}^{t_{i}}\left(t_{i}-s\right)^{q-2} d s \\
& +\mu_{2} \sum_{i=1}^{n}\left|G_{1 s}^{\prime}\left(t, t_{i}\right)\right|+\mu_{3} \sum_{i=1}^{n}\left|G_{1}\left(t, t_{i}\right)\right| \\
& =\frac{\gamma \mu_{1}}{\Gamma(q+1)}+\frac{\gamma \mu_{1}}{\Gamma(q+1)} \sum_{i=1}^{n+1}\left|G_{1 s}^{\prime}\left(t, t_{i}\right)\right|+\frac{\gamma \mu_{1}}{\Gamma(q)} \sum_{i=1}^{n}\left|G_{1}\left(t, t_{i}\right)\right| \\
& +\mu_{2} \sum_{i=1}^{n}\left|G_{1 s}^{\prime}\left(t, t_{i}\right)\right|+\mu_{3} \sum_{i=1}^{n}\left|G_{1}\left(t, t_{i}\right)\right| \\
& \leq \gamma \mu_{1}\left[\frac{1}{\Gamma(q+1)}+\frac{1}{\Gamma(q+1)}(n+1) c_{2}+\frac{1}{\Gamma(q)} n c_{1}\right] \\
& +n\left(c_{2} \mu_{2}+c_{1} \mu_{3}\right):=\mu \text {, }
\end{aligned}
$$

where

$$
\gamma=\max _{t \in J} \omega(t)
$$

Furthermore, for any $t \in\left(t_{k}, t_{k+1}\right], 0 \leq k \leq n$, we obtain

$$
\begin{aligned}
\left|(T x)^{\prime}(t)\right| \leq & \int_{t_{k}}^{t} \frac{(t-s)^{q-2}}{\Gamma(q-1)} \omega(s)\left|f\left(s, x(s), x^{\prime}(s)\right)\right| d s \\
& +\sum_{i=1}^{n+1}\left|G_{1 s t}^{\prime \prime}\left(t, t_{i}\right)\right| \int_{t_{i-1}}^{t_{i}} \frac{\left(t_{i}-s\right)^{q-1}}{\Gamma(q)} \omega(s)\left|f\left(s, x(s), x^{\prime}(s)\right)\right| d s \\
& +\sum_{i=1}^{n}\left|G_{1 t}^{\prime}\left(t, t_{i}\right)\right| \int_{t_{i-1}}^{t_{i}} \frac{\left(t_{i}-s\right)^{q-2}}{\Gamma(q-1)} \omega(s)\left|f\left(s, x(s), x^{\prime}(s)\right)\right| d s \\
& +\sum_{i=1}^{n}\left|G_{1 s t}^{\prime \prime}\left(t, t_{i}\right)\right|\left|\mathcal{I}_{i}\left(x\left(t_{i}\right)\right)\right|+\sum_{i=1}^{n}\left|G_{1 t}^{\prime}\left(t, t_{i}\right)\right|\left|\mathcal{J}_{i}\left(x\left(t_{i}\right)\right)\right| \\
\leq & \frac{\gamma \mu_{1}}{\Gamma(q-1)} \int_{t_{k}}^{t}(t-s)^{q-2} d s+\frac{\gamma \mu_{1}}{\Gamma(q)} \sum_{i=1}^{n+1}\left|G_{1 s t}^{\prime \prime}\left(t, t_{i}\right)\right| \int_{t_{i-1}}^{t_{i}}\left(t_{i}-s\right)^{q-1} d s \\
& +\frac{\gamma \mu_{1}}{\Gamma(q-1)} \sum_{i=1}^{n}\left|G_{1 t}^{\prime}\left(t, t_{i}\right)\right| \int_{t_{i-1}}^{t_{i}}\left(t_{i}-s\right)^{q-2} d s \\
& +\mu_{2} \sum_{i=1}^{n}\left|G_{1 s t}^{\prime \prime}\left(t, t_{i}\right)\right|+\mu_{3} \sum_{i=1}^{n}\left|G_{1 t}^{\prime}\left(t, t_{i}\right)\right|
\end{aligned}
$$




$$
\begin{aligned}
= & \frac{\gamma \mu_{1}}{\Gamma(q)}+\frac{\gamma \mu_{1}}{\Gamma(q+1)} \sum_{i=1}^{n+1}\left|G_{1 s t}^{\prime \prime}\left(t, t_{i}\right)\right|+\frac{\gamma \mu_{1}}{\Gamma(q)} \sum_{i=1}^{n}\left|G_{1 t}^{\prime}\left(t, t_{i}\right)\right| \\
& +\mu_{2} \sum_{i=1}^{n}\left|G_{1 s t}^{\prime \prime}\left(t, t_{i}\right)\right|+\mu_{3} \sum_{i=1}^{n}\left|G_{1 t}^{\prime}\left(t, t_{i}\right)\right| \\
\leq & \gamma \mu_{1}\left[\frac{1}{\Gamma(q)}+\frac{1}{\Gamma(q+1)}(n+1) c_{3}+\frac{1}{\Gamma(q)} n c_{2}\right]+n c_{3} \mu_{2}+n c_{2} \mu_{3}:=\bar{\mu} .
\end{aligned}
$$

On the other hand, from (3.5), for $t_{1}, t_{2} \in\left(t_{k}, t_{k+1}\right]$ with $t_{1}<t_{2}$, we have

$$
\left|(T x)^{\prime}\left(t_{2}\right)-(T x)^{\prime}\left(t_{1}\right)\right| \leq \int_{t_{1}}^{t_{2}}\left|(T x)^{\prime}(s)\right| d s \leq \bar{\mu}\left(t_{2}-t_{1}\right) .
$$

It follows from (3.3), (3.5) and (3.6) that $T$ is equicontinuous on all subintervals $\left(t_{k}, t_{k+1}\right]$, $k=1,2, \ldots, n$. Thus, by the Arzela-Ascoli theorem, the operator $T: P C^{1}[J, R] \rightarrow P C^{1}[J, R]$ is completely continuous.

To prove our main results, we also need the following two lemmas.

Lemma 3.3 (See $[47,48]$ ) (Schauder fixed point theorem) Let D be a nonempty, closed, bounded, convex subset of a B-space $X$, and suppose that $T: D \rightarrow D$ is a completely continuous operator. Then $T$ has a fixed point $x \in D$.

Lemma 3.4 (See $[47,48]$ ) (Leray-Schauder fixed point theorem) Let $X$ be a real Banach space and $T: X \rightarrow X$ be a completely continuous operator. If

$$
\{x: x \in X, x=\lambda T x, 0<\lambda<1\}
$$

is bounded, then $T$ has a fixed point $x^{*} \in \Omega$, where

$$
\Omega=\{x: x \in X,\|x\| \leq l\}, \quad l=\sup \{x: x \in X, x=\lambda T x, 0<\lambda<1\} .
$$

\section{Existence of solutions}

In this section, we apply Lemma 3.3, Lemma 3.4 and the contraction mapping principle to establish the existence of solutions of problem (1.1). Let us begin by introducing some notation. Define

$$
\begin{aligned}
& \xi=\lim _{|x|+|y| \rightarrow \infty}\left(\max _{t \in J} \frac{|f(t, x, y)|}{|x|+|y|}\right), \\
& \xi_{1}=\lim _{|x| \rightarrow \infty} \frac{\left|\mathrm{I}_{k}(x)\right|}{|x|}, \quad \xi_{2}=\lim _{|x| \rightarrow \infty} \frac{\left|\mathcal{J}_{k}(x)\right|}{|x|}, \quad k=1,2, \ldots, n .
\end{aligned}
$$

Theorem 4.1 Assume that $\left(\mathrm{H}_{1}\right)-\left(\mathrm{H}_{3}\right)$ hold. Suppose further that

$$
\delta=\max \left\{\delta_{1}, \delta_{2}\right\}<1,
$$

where

$$
\delta_{1}=\gamma\left[\frac{2 \xi\left(1+(n+1) c_{2}\right)}{\Gamma(q+1)}+\frac{2 \xi n c_{1}}{\Gamma(q)}\right]+n\left(\xi_{1} c_{2}+\xi_{2} c_{1}\right)
$$


and

$$
\delta_{2}=\gamma\left[\frac{2 \xi\left(1+n c_{2}\right)}{\Gamma(q)}+\frac{2 \xi(n+1) c_{3}}{\Gamma(q+1)}\right]+n\left(\xi_{1} c_{3}+\xi_{2} c_{2}\right)
$$

here $\gamma$ is defined in (3.4). Then problem (1.1) has at least one solution $x \in P C^{1}[J, R] \cap$ $C^{2}\left[J^{\prime}, R\right]$.

Proof We shall use Schauder's fixed point theorem to prove that $T$ has a fixed point. First, recall that the operator $T: P C^{1}[J, R] \rightarrow P C^{1}[J, R]$ is completely continuous (see the proof of Lemma 3.2).

On account of (4.1), we can choose $\xi^{\prime}>\xi, \xi_{1}^{\prime}>\xi_{1}$ and $\xi_{2}^{\prime}>\xi_{2}$ such that

$$
\delta_{1}^{\prime}=\gamma\left[\frac{2 \xi^{\prime}\left(1+(n+1) c_{2}\right)}{\Gamma(q+1)}+\frac{2 \xi^{\prime} n c_{1}}{\Gamma(q)}\right]+n\left(\xi_{1}^{\prime} c_{2}+\xi_{2}^{\prime} c_{1}\right)<1
$$

and

$$
\delta_{2}^{\prime}=\gamma\left[\frac{2 \xi^{\prime}\left(1+n c_{2}\right)}{\Gamma(q)}+\frac{2 \xi^{\prime}(n+1) c_{3}}{\Gamma(q+1)}\right]+n\left(\xi_{1}^{\prime} c_{3}+\xi_{2}^{\prime} c_{2}\right)<1
$$

By the definition of $\xi$, there exists $l>0$ such that

$$
|f(t, x, y)|<\xi^{\prime}(|x|+|y|), \quad \forall t \in J,|x|+|y|>l,
$$

so

$$
|f(t, x, y)|<\xi^{\prime}(|x|+|y|)+M, \quad \forall t \in J, x, y \in R,
$$

where

$$
M=\max _{t \in J,|x|+|y| \leq l}|f(t, x, y)|<+\infty .
$$

Similarly, we have

$$
\left|\mathcal{I}_{k}(x)\right|<\xi_{1}^{\prime}|x|+M_{k}, \quad \forall x \in R, k=1,2, \ldots, n,
$$

and

$$
\left|\mathcal{J}_{k}(x)\right| \leq \xi_{2}^{\prime}|x|+\bar{M}_{k}, \quad \forall x \in R, k=1,2, \ldots, n,
$$

where $M_{k}, \bar{M}_{k}$ are positive constants.

It follows from (3.2) and (4.4)-(4.6) that

$$
\begin{aligned}
|(T x)(t)| \leq & \int_{t_{k}}^{t} \frac{(t-s)^{q-1}}{\Gamma(q)} \omega(s)|f(s, x(s), y(s))| d s \\
& +\sum_{i=1}^{n+1}\left|G_{1 s}^{\prime}\left(t, t_{i}\right)\right| \int_{t_{i-1}}^{t_{i}} \frac{\left(t_{i}-s\right)^{q-1}}{\Gamma(q)} \omega(s)|f(s, x(s), y(s))| d s
\end{aligned}
$$




$$
\begin{aligned}
& +\sum_{i=1}^{n}\left|G_{1}\left(t, t_{i}\right)\right| \int_{t_{i-1}}^{t_{i}} \frac{\left(t_{i}-s\right)^{q-2}}{\Gamma(q-1)} \omega(s)|f(s, x(s), y(s))| d s \\
& +\sum_{i=1}^{n}\left|G_{1 s}^{\prime}\left(t, t_{i}\right)\right|\left|\mathcal{I}_{i}\left(x\left(t_{i}\right)\right)\right|+\sum_{i=1}^{n}\left|G_{1}\left(t, t_{i}\right)\right|\left|\mathcal{J}_{i}\left(x\left(t_{i}\right)\right)\right| \\
\leq & \gamma\left(\xi^{\prime}(|x|+|y|)+M\right) \int_{t_{k}}^{t} \frac{(t-s)^{q-1}}{\Gamma(q)} d s \\
& +\gamma\left(\xi^{\prime}(|x|+|y|)+M\right) \sum_{i=1}^{n+1}\left|G_{1 s}^{\prime}\left(t, t_{i}\right)\right| \int_{t_{i-1}}^{t_{i}} \frac{\left(t_{i}-s\right)^{q-1}}{\Gamma(q)} d s \\
& +\gamma\left(\xi^{\prime}(|x|+|y|)+M\right) \sum_{i=1}^{n}\left|G_{1}\left(t, t_{i}\right)\right| \int_{t_{i-1}}^{t_{i}} \frac{\left(t_{i}-s\right)^{q-2}}{\Gamma(q-1)} d s \\
& +\left(\xi_{1}^{\prime}|x|+M_{k}\right) \sum_{i=1}^{n}\left|G_{1 s}^{\prime}\left(t, t_{i}\right)\right|+\left(\xi_{2}^{\prime}|x|+\bar{M}_{k}\right) \sum_{i=1}^{n}\left|G_{1}\left(t, t_{i}\right)\right| \\
\leq & \gamma\left[\frac{\xi^{\prime}(|x|+|y|)+M}{\Gamma(q+1)}+\frac{\xi^{\prime}(|x|+|y|)+M}{\Gamma(q+1)}(n+1) c_{2}+\frac{\xi^{\prime}(|x|+|y|)+M}{\Gamma(q)} n c_{1}\right] \\
& +\left(\xi_{1}^{\prime}|x|+M_{k}\right) n c_{2}+\left(\xi_{2}^{\prime}|x|+\bar{M}_{k}\right) n c_{1} \\
\leq & \gamma\left[\frac{2 \xi^{\prime}\|x\|_{P C^{1}}+M}{\Gamma(q+1)}+\frac{2 \xi^{\prime}\|x\|_{P C^{1}}+M}{\Gamma(q+1)}(n+1) c_{2}+\frac{2 \xi^{\prime}\|x\|_{P C^{1}}+M}{\Gamma(q)} n c_{1}\right] \\
& +\left(\xi_{1}^{\prime}\|x\|_{P C^{1}}+M_{k}\right) n c_{2}+\left(\xi_{2}^{\prime}\|x\|_{P C^{1}}+\bar{M}_{k}\right) n c_{1} \\
= & \delta_{1}^{\prime}\|x\|_{P C^{1}}+M^{(1)},
\end{aligned}
$$

where $\delta_{1}^{\prime}$ is defined by (4.2) and $M^{(1)}$ is defined by

$$
M^{(1)}=\gamma\left[\frac{M\left(1+(n+1) c_{2}\right)}{\Gamma(q+1)}+\frac{M n c_{1}}{\Gamma(q)}\right]+n\left(c_{2} M_{k}+c_{1} \bar{M}_{k}\right) .
$$

Similarly, from (3.2) and (4.4)-(4.6), we get

$$
\begin{aligned}
\left|(T x)^{\prime}(t)\right| \leq & \int_{t_{k}}^{t} \frac{(t-s)^{q-2}}{\Gamma(q-1)} \omega(s)\left|f\left(s, x(s), x^{\prime}(s)\right)\right| d s \\
& +\sum_{i=1}^{n+1}\left|G_{1 s t}^{\prime \prime}\left(t, t_{i}\right)\right| \int_{t_{i-1}}^{t_{i}} \frac{\left(t_{i}-s\right)^{q-1}}{\Gamma(q)} \omega(s)\left|f\left(s, x(s), x^{\prime}(s)\right)\right| d s \\
& +\sum_{i=1}^{n}\left|G_{1 t}^{\prime}\left(t, t_{i}\right)\right| \int_{t_{i-1}}^{t_{i}} \frac{\left(t_{i}-s\right)^{q-2}}{\Gamma(q-1)} \omega(s)\left|f\left(s, x(s), x^{\prime}(s)\right)\right| d s \\
& +\sum_{i=1}^{n}\left|G_{1 s t}^{\prime \prime}\left(t, t_{i}\right)\right|\left|\mathcal{I}_{i}\left(x\left(t_{i}\right)\right)\right|+\sum_{i=1}^{n}\left|G_{1 t}^{\prime}\left(t, t_{i}\right)\right|\left|\mathcal{J}_{i}\left(x\left(t_{i}\right)\right)\right| \\
\leq & \gamma\left(\xi^{\prime}(|x|+|y|)+M\right) \int_{t_{k}}^{t} \frac{(t-s)^{q-2}}{\Gamma(q-1)} d s \\
& +\gamma\left(\xi^{\prime}(|x|+|y|)+M\right) \sum_{i=1}^{n+1}\left|G_{1 s t}^{\prime \prime}\left(t, t_{i}\right)\right| \int_{t_{i-1}}^{t_{i}} \frac{\left(t_{i}-s\right)^{q-1}}{\Gamma(q)} d s
\end{aligned}
$$




$$
\begin{aligned}
& +\gamma\left(\xi^{\prime}(|x|+|y|)+M\right) \sum_{i=1}^{n}\left|G_{1 t}^{\prime}\left(t, t_{i}\right)\right| \int_{t_{i-1}}^{t_{i}} \frac{\left(t_{i}-s\right)^{q-2}}{\Gamma(q-1)} d s \\
& +\left(\xi_{1}^{\prime}|x|+M_{k}\right) \sum_{i=1}^{n}\left|G_{1 s t}^{\prime \prime}\left(t, t_{i}\right)\right|+\left(\xi_{2}^{\prime}|x|+\bar{M}_{k}\right) \sum_{i=1}^{n}\left|G_{1 t}^{\prime}\left(t, t_{i}\right)\right| \\
\leq & \gamma\left(2 \xi^{\prime}\|x\|_{P C^{1}}+M\right) \int_{t_{k}}^{t} \frac{(t-s)^{q-2}}{\Gamma(q-1)} d s \\
& +\gamma\left(2 \xi^{\prime}\|x\|_{P C^{1}}+M\right) \sum_{i=1}^{n+1}\left|G_{1 s t}^{\prime \prime}\left(t, t_{i}\right)\right| \int_{t_{i-1}}^{t_{i}} \frac{\left(t_{i}-s\right)^{q-1}}{\Gamma(q)} d s \\
& +\gamma\left(2 \xi^{\prime}\|x\|_{P C^{1}}+M\right) \sum_{i=1}^{n}\left|G_{1 t}^{\prime}\left(t, t_{i}\right)\right| \int_{t_{i-1}}^{t_{i}} \frac{\left(t_{i}-s\right)^{q-2}}{\Gamma(q-1)} d s \\
& +\left(\xi_{1}^{\prime}\|x\|_{P C^{1}}+M_{k}\right) \sum_{i=1}^{n}\left|G_{1 s t}^{\prime \prime}\left(t, t_{i}\right)\right|+\left(\xi_{2}^{\prime}\|x\|_{P C^{1}}+\bar{M}_{k}\right) \sum_{i=1}^{n}\left|G_{1 t}^{\prime}\left(t, t_{i}\right)\right| \\
\leq & \gamma\left[\frac{2 \xi^{\prime}\|x\|_{P C^{1}}+M}{\Gamma(q)}+\frac{2 \xi^{\prime}\|x\|_{P C^{1}}+M}{\Gamma(q+1)}(n+1) c_{3}+\frac{2 \xi^{\prime}\|x\|_{P C^{1}}+M}{\Gamma(q)} n c_{2}\right] \\
& +\left(\xi_{1}^{\prime}\|x\|_{P C^{1}}+M_{k}\right) n c_{3}+\left(\xi_{2}^{\prime}\|x\|_{P C^{1}}+\bar{M}_{k}\right) n c_{2} \\
= & \delta_{2}^{\prime}\|x\|_{P C^{1}}+M^{(2)},
\end{aligned}
$$

where $\delta_{1}^{\prime}$ is defined by (4.3) and $M^{(2)}$ is defined by

$$
M^{(2)}=\gamma\left[\frac{M\left(1+n c_{2}\right)}{\Gamma(q)}+\frac{(n+1) c_{3} M}{\Gamma(q+1)}\right]+n\left(M_{k} c_{3}+\bar{M}_{k} c_{2}\right) .
$$

It follows from (4.7) and (4.8) that

$$
\|T x\|_{P C^{1}} \leq \delta^{\prime}\|x\|_{P C^{1}}+M^{\prime}, \quad \forall x \in P C^{1}[J, E]
$$

where

$$
\delta^{\prime}=\max \left\{\delta_{1}^{\prime}, \delta_{2}^{\prime}\right\}<1, \quad M^{\prime}=\max \left\{M^{(1)}, M^{(2)}\right\} .
$$

Hence, we can choose a sufficiently large $r>0$ such that $T\left(B_{r}\right) \subset B_{r}$, where

$$
B_{r}=\left\{x \in P C^{1}:\|x\|_{P C^{1}} \leq r\right\} .
$$

Consequently, Lemma 3.3 implies that $T$ has a fixed point in $B_{r}$, and the proof is complete.

Remark 4.1 Condition (4.1) is certainly satisfied if $|f(t, x, y)| /|x|+|y| \rightarrow 0$ uniformly in $t \in J$ as $|x|+|y| \rightarrow+\infty,\left|\mathcal{I}_{k}(x)\right| /|x| \rightarrow 0$ as $|x| \rightarrow+\infty$ and $\left|\mathcal{J}_{k}(x)\right| /|x| \rightarrow 0$ as $|x| \rightarrow+\infty$ $(k=1,2, \ldots, n)$.

Theorem 4.2 Assume that $\left(\mathrm{H}_{1}\right)-\left(\mathrm{H}_{3}\right)$ hold.In addition, let $f$, $\mathcal{I}_{k}$ and $\mathcal{J}_{k}$ satisfy the following conditions: 
$\left(\mathrm{H}_{4}\right)$ There exists a nonnegative function $v_{1}(t) \in C[0,1]$ with $v_{1}(t)>0$ on a subinterval of $[0,1]$ such that

$$
|f(t, x, y)| \leq v_{1}(t), \quad \forall(t, x, y) \in J \times R \times R .
$$

$\left(\mathrm{H}_{5}\right)$ There exist constants $v_{2}, v_{3}>0$ such that

$$
\left|\mathcal{I}_{k}(x)\right|<\nu_{2}, \quad\left|\mathcal{J}_{k}(x)\right|<\nu_{3}
$$

for all $x \in R, k=1,2, \ldots, n$.

Then problem (1.1) has at least one solution.

Proof It follows from Lemma 3.2 that the operator $T: P C^{1}[, R] \rightarrow P C^{1}[, R]$ is completely continuous.

Next, we show that the set

$$
V=\left\{x \in P C^{1}(J, R) \mid x=\lambda T x, 0<\lambda<1\right\}
$$

is bounded.

Let $x \in V$. Then $x=\lambda T x$ for $0<\lambda<1$. For any $t \in J$, we have

$$
\begin{aligned}
x(t)= & \lambda\left[\int_{t_{k}}^{t} \frac{(t-s)^{q-1}}{\Gamma(q)} \omega(s) f\left(s, x(s), x^{\prime}(s)\right) d s\right. \\
& +\sum_{i=1}^{n+1} G_{1 s}^{\prime}\left(t, t_{i}\right) \int_{t_{i-1}}^{t_{i}} \frac{\left(t_{i}-s\right)^{q-1}}{\Gamma(q)} \omega(s) f\left(s, x(s), x^{\prime}(s)\right) d s \\
& -\sum_{i=1}^{n} G_{1}\left(t, t_{i}\right) \int_{t_{i-1}}^{t_{i}} \frac{\left(t_{i}-s\right)^{q-2}}{\Gamma(q-1)} \omega(s) f\left(s, x(s), x^{\prime}(s)\right) d s \\
& \left.+\sum_{i=1}^{n} G_{1 s}^{\prime}\left(t, t_{i}\right) \mathcal{I}_{i}\left(x\left(t_{i}\right)\right)-\sum_{i=1}^{n} G_{1}\left(t, t_{i}\right) \mathcal{J}_{i}\left(x\left(t_{i}\right)\right)\right] .
\end{aligned}
$$

It follows from $\left(\mathrm{H}_{4}\right),\left(\mathrm{H}_{5}\right),(3.2)$ and (4.9) that

$$
\begin{aligned}
|x(t)|= & \lambda|(T x)(t)| \\
\leq & \int_{t_{k}}^{t} \frac{(t-s)^{q-1}}{\Gamma(q)} \omega(s)|f(s, x(s), y(s))| d s \\
& +\sum_{i=1}^{n+1}\left|G_{1 s}^{\prime}\left(t, t_{i}\right)\right| \int_{t_{i-1}}^{t_{i}} \frac{\left(t_{i}-s\right)^{q-1}}{\Gamma(q)} \omega(s)|f(s, x(s), y(s))| d s \\
& +\sum_{i=1}^{n}\left|G_{1}\left(t, t_{i}\right)\right| \int_{t_{i-1}}^{t_{i}} \frac{\left(t_{i}-s\right)^{q-2}}{\Gamma(q-1)} \omega(s)|f(s, x(s), y(s))| d s \\
& +\sum_{i=1}^{n}\left|G_{1 s}^{\prime}\left(t, t_{i}\right)\right|\left|\mathcal{I}_{i}\left(x\left(t_{i}\right)\right)\right|+\sum_{i=1}^{n}\left|G_{1}\left(t, t_{i}\right)\right|\left|\mathcal{J}_{i}\left(x\left(t_{i}\right)\right)\right|
\end{aligned}
$$


where

$$
\begin{aligned}
& \leq \gamma \tau \int_{t_{k}}^{t} \frac{(t-s)^{q-1}}{\Gamma(q)} d s+\gamma \tau \sum_{i=1}^{n+1}\left|G_{1 s}^{\prime}\left(t, t_{i}\right)\right| \int_{t_{i-1}}^{t_{i}} \frac{\left(t_{i}-s\right)^{q-1}}{\Gamma(q)} d s \\
& \quad+\gamma \tau \sum_{i=1}^{n}\left|G_{1}\left(t, t_{i}\right)\right| \int_{t_{i-1}}^{t_{i}} \frac{\left(t_{i}-s\right)^{q-2}}{\Gamma(q-1)} d s \\
& \quad+v_{2} \sum_{i=1}^{n}\left|G_{1 s}^{\prime}\left(t, t_{i}\right)\right|+v_{3} \sum_{i=1}^{n}\left|G_{1}\left(t, t_{i}\right)\right| \\
& \leq \gamma \tau\left[\frac{1}{\Gamma(q+1)}+\frac{1}{\Gamma(q+1)}(n+1) c_{2}+\frac{1}{\Gamma(q)} n c_{1}\right]+v_{2} n c_{2}+v_{3} n c_{1},
\end{aligned}
$$

$$
\tau=\max _{t \in J} v_{1}(t)
$$

It follows from (4.10) that

$$
\|x\|_{P C} \leq \gamma \tau\left[\frac{1+c_{2}+n c_{2}}{\Gamma(q+1)}+\frac{n c_{1}}{\Gamma(q)}\right]+n\left(v_{2} c_{2}+v_{3} c_{1}\right)
$$

Furthermore, for any $t \in\left(t_{k}, t_{k+1}\right], 0 \leq k \leq n$, we obtain

$$
\begin{aligned}
\left|x^{\prime}(t)\right|= & \lambda\left|(T x)^{\prime}(t)\right| \\
\leq & \int_{t_{k}}^{t} \frac{(t-s)^{q-2}}{\Gamma(q-1)} \omega(s)\left|f\left(s, x(s), x^{\prime}(s)\right)\right| d s \\
& +\sum_{i=1}^{n+1}\left|G_{1 s t}^{\prime \prime}\left(t, t_{i}\right)\right| \int_{t_{i-1}}^{t_{i}} \frac{\left(t_{i}-s\right)^{q-1}}{\Gamma(q)} \omega(s)\left|f\left(s, x(s), x^{\prime}(s)\right)\right| d s \\
& +\sum_{i=1}^{n}\left|G_{1 t}^{\prime}\left(t, t_{i}\right)\right| \int_{t_{i-1}}^{t_{i}} \frac{\left(t_{i}-s\right)^{q-2}}{\Gamma(q-1)} \omega(s)\left|f\left(s, x(s), x^{\prime}(s)\right)\right| d s \\
& +\sum_{i=1}^{n}\left|G_{1 s t}^{\prime \prime}\left(t, t_{i}\right)\right|\left|\mathcal{I}_{i}\left(x\left(t_{i}\right)\right)\right|+\sum_{i=1}^{n}\left|G_{1 t}^{\prime}\left(t, t_{i}\right)\right|\left|\mathcal{J}_{i}\left(x\left(t_{i}\right)\right)\right| \\
\leq & \gamma \tau \int_{t_{k}}^{t} \frac{(t-s)^{q-2}}{\Gamma(q-1)} d s+\gamma \tau \sum_{i=1}^{n+1}\left|G_{1 s t}^{\prime \prime}\left(t, t_{i}\right)\right| \int_{t_{i-1}}^{t_{i}} \frac{\left(t_{i}-s\right)^{q-1}}{\Gamma(q)} d s \\
& +\gamma \tau \sum_{i=1}^{n}\left|G_{1 t}^{\prime}\left(t, t_{i}\right)\right| \int_{t_{i-1}}^{t_{i}} \frac{\left(t_{i}-s\right)^{q-2}}{\Gamma(q-1)} d s+v_{2} \sum_{i=1}^{n}\left|G_{1 s t}^{\prime \prime}\left(t, t_{i}\right)\right|+v_{3} \sum_{i=1}^{n}\left|G_{1 t}^{\prime}\left(t, t_{i}\right)\right| \\
\leq & \gamma \tau\left[\frac{1}{\Gamma(q)}+\frac{1}{\Gamma(q+1)}(n+1) c_{3}+\frac{1}{\Gamma(q)} n c_{2}\right]+v_{2} n c_{3}+v_{3} n c_{2},
\end{aligned}
$$

which, for any $t \in J$, yields

$$
\left\|x^{\prime}\right\|_{P C} \leq \gamma \tau\left[\frac{1+n c_{2}}{\Gamma(q)}+\frac{(n+1) c_{3}}{\Gamma(q+1)}\right]+n\left(v_{2} c_{3}+v_{3} c_{2}\right) .
$$

It follows from (4.11) and (4.12) that

$$
\|x\|_{P C^{1}} \leq \zeta, \quad \forall x \in P C^{1}(J, R),
$$


where $\zeta \leq \max \left\{\zeta_{1}, \zeta_{2}\right\}$, here

$$
\begin{aligned}
& \zeta_{1}=\gamma \tau\left[\frac{1+c_{2}+n c_{2}}{\Gamma(q+1)}+\frac{n c_{1}}{\Gamma(q)}\right]+n\left(v_{2} c_{2}+v_{3} c_{1}\right), \\
& \zeta_{2}=\gamma \tau\left[\frac{1+n c_{2}}{\Gamma(q)}+\frac{(n+1) c_{3}}{\Gamma(q+1)}\right]+n\left(v_{2} c_{3}+v_{3} c_{2}\right) .
\end{aligned}
$$

So it follows from (4.13) that the set $V$ is bounded. Thus, as a consequence of Lemma 3.4, the operator $T$ has at least one fixed point. Consequently, the problem (1.1) has at least one solution. This finishes the proof.

Finally we consider the existence of a unique solution for problem (1.1) by applying the contraction mapping principle.

Theorem 4.3 Assume that $\left(\mathrm{H}_{1}\right)-\left(\mathrm{H}_{3}\right)$ hold.In addition, let $f$, $\mathcal{I}_{k}$ and $\mathcal{J}_{k}$ satisfy the following conditions:

$\left(\mathrm{H}_{6}\right)$ There exists a constant $L_{1}>0$ such that

$$
|f(t, x, y)-f(t, \bar{x}, \bar{y})| \leq L_{1}(|x-\bar{x}|+|y-\bar{y}|)
$$

for each $t \in J$ and all $x, y, \bar{x}, \bar{y} \in R$.

$\left(\mathrm{H}_{7}\right)$ There exist constants $L_{2}, L_{3}>0$ such that

$$
\left|\mathcal{I}_{k}(x)-\mathcal{I}_{k}(y)\right|<L_{2}|x-y|, \quad\left|\mathcal{J}_{k}(x)-\mathcal{I}_{k}(y)\right|<L_{3}|x-y|
$$

for all $x, y \in R, k=1,2, \ldots, n$.

If

$$
\Lambda<\max \left\{\Lambda_{1}, \Lambda_{2}\right\}<1,
$$

where

$$
\begin{aligned}
& \Lambda_{1}=\frac{2 L_{1}\left(1+c_{1}+n c_{1}\right)}{\Gamma(q+1)} \gamma+\frac{2 L_{1} n c_{1}}{\Gamma(q)} \gamma+n\left(c_{2} L_{2}+c_{1} L_{3}\right), \\
& \Lambda_{2}=\frac{2 L_{1}\left(1+n c_{2}\right)}{\Gamma(q)} \gamma+\frac{2 L_{1}(n+1) c_{3}}{\Gamma(q+1)} \gamma+n\left(c_{3} L_{2}+c_{2} L_{3}\right),
\end{aligned}
$$

here $c_{1}$ and $c_{2}$ are defined in (2.10), then problem (1.1) has a unique solution.

Proof Let $x, y \in P C^{1}[J, R]$. Then, for each $t \in J$, it follows from $\left(\mathrm{H}_{6}\right),\left(\mathrm{H}_{7}\right)$ and (3.2) that

$$
\begin{aligned}
& |(T x)(t)-(T y)(t)| \\
& \quad \leq \int_{t_{k}}^{t} \frac{(t-s)^{q-1}}{\Gamma(q)} \omega(s)|f(s, x(s), \bar{x}(s))-f(s, y(s), \bar{y}(s))| d s \\
& \quad+\sum_{i=1}^{n+1}\left|G_{1 s}^{\prime}\left(t, t_{i}\right)\right| \int_{t_{i-1}}^{t_{i}} \frac{\left(t_{i}-s\right)^{q-1}}{\Gamma(q)} \omega(s)|f(s, x(s), \bar{x}(s))-f(s, y(s), \bar{y}(s))| d s
\end{aligned}
$$




$$
\begin{aligned}
& +\sum_{i=1}^{n}\left|G_{1}\left(t, t_{i}\right)\right| \int_{t_{i-1}}^{t_{i}} \frac{\left(t_{i}-s\right)^{q-2}}{\Gamma(q-1)} \omega(s)|f(s, x(s), \bar{x}(s))-f(s, y(s), \bar{y}(s))| d s \\
& +\sum_{i=1}^{n}\left|G_{1 s}^{\prime}\left(t, t_{i}\right)\right|\left|\mathcal{I}_{i}\left(x\left(t_{i}\right)\right)-\mathcal{I}_{i}\left(y\left(t_{i}\right)\right)\right|+\sum_{i=1}^{n}\left|G_{1}\left(t, t_{i}\right)\right|\left|\mathcal{J}_{i}\left(x\left(t_{i}\right)\right)-\mathcal{J}_{i}\left(y\left(t_{i}\right)\right)\right| \\
\leq & \frac{2 L_{1}\|x-y\|_{P C^{1}}}{\Gamma(q)} \gamma \int_{t_{k}}^{t}(t-s)^{q-1} d s+\frac{2 L_{1}\|x-y\|_{P C^{1}}}{\Gamma(q)} \gamma \sum_{i=1}^{n+1}\left|G_{1}\left(t, t_{i}\right)\right| \int_{t_{i-1}}^{t_{i}}\left(t_{i}-s\right)^{q-2} d s \\
& +\frac{2 L_{1}\|x-y\|_{P C^{1}}}{\Gamma(q-1)} \gamma \sum_{i=1}^{n}\left|G_{1 s}^{\prime}\left(t, t_{i}\right)\right| \int_{t_{i-1}}^{t_{i}}\left(t_{i}-s\right)^{q-1} d s \\
& +L_{2}\|x-y\|_{P C^{1}} \sum_{i=1}^{n}\left|G_{1 s}^{\prime}\left(t, t_{i}\right)\right|+L_{3}\|x-y\|_{P C^{1}} \sum_{i=1}^{n}\left|G_{1}\left(t, t_{i}\right)\right| \\
\leq & \frac{2 L_{1}\|x-y\|_{P C^{1}}}{\Gamma(q+1)} \gamma+\frac{2 L_{1}\|x-y\|_{P C^{1}}}{\Gamma(q+1)} \gamma(n+1) c_{1}+\frac{2 L_{1}\|x-y\|_{P C^{1}}}{\Gamma(q)} \gamma n c_{2} \\
& +n c_{2} L_{2}\|x-y\|_{P C^{1}}+n c_{1} L_{3}\|x-y\|_{P C^{1}} \\
= & {\left[\frac{2 L_{1}\left(1+c_{1}+n c_{1}\right)}{\Gamma(q+1)} \gamma+\frac{2 L_{1} n c_{1}}{\Gamma(q)} \gamma+n\left(c_{2} L_{2}+c_{1} L_{3}\right)\right]\|x-y\|_{P C^{1}} }
\end{aligned}
$$

and

$$
\begin{aligned}
\left|(T x)^{\prime}(t)-(T y)^{\prime}(t)\right| & \\
\leq & \int_{t_{k}}^{t} \frac{(t-s)^{q-2}}{\Gamma(q-1)} \omega(s)|f(s, x(s), \bar{x}(s))-f(s, y(s), \bar{y}(s))| d s \\
& +\sum_{i=1}^{n+1}\left|G_{1 s t}^{\prime \prime}\left(t, t_{i}\right)\right| \int_{t_{i-1}}^{t_{i}} \frac{\left(t_{i}-s\right)^{q-1}}{\Gamma(q)} \omega(s)|f(s, x(s), \bar{x}(s))-f(s, y(s), \bar{y}(s))| d s \\
& +\sum_{i=1}^{n}\left|G_{1 t}^{\prime}\left(t, t_{i}\right)\right| \int_{t_{i-1}}^{t_{i}} \frac{\left(t_{i}-s\right)^{q-2}}{\Gamma(q-1)} \omega(s)|f(s, x(s), \bar{x}(s))-f(s, y(s), \bar{y}(s))| d s \\
& +\sum_{i=1}^{n}\left|G_{1 s t}^{\prime \prime}\left(t, t_{i}\right)\right|\left|\mathcal{I}_{i}\left(x\left(t_{i}\right)\right)-\mathcal{I}_{i}\left(y\left(t_{i}\right)\right)\right|+\sum_{i=1}^{n}\left|G_{1 t}^{\prime}\left(t, t_{i}\right)\right|\left|\mathcal{J}_{i}\left(x\left(t_{i}\right)\right)-\mathcal{J}_{i}\left(y\left(t_{i}\right)\right)\right| \\
\leq & \frac{2 L_{1}\|x-y\|_{P C^{1}}}{\Gamma(q-1)} \gamma \int_{t_{k}}^{t}(t-s)^{q-2} d s+\frac{2 L_{1}\|x-y\|_{P C^{1}}}{\Gamma(q)} \gamma \sum_{i=1}^{n+1}\left|G_{1 s t}^{\prime \prime}\left(t, t_{i}\right)\right| \int_{t_{i-1}}^{t_{i}}\left(t_{i}-s\right)^{q-1} d s \\
& +\frac{2 L_{1}\|x-y\|_{P C^{1}}}{\Gamma(q-1)} \gamma \sum_{i=1}^{n}\left|G_{1 t}^{\prime}\left(t, t_{i}\right)\right| \int_{t_{i-1}}^{t_{i}}\left(t_{i}-s\right)^{q-2} d s \\
& +L_{2}\|x-y\|_{P C^{1}} \sum_{i=1}^{n}\left|G_{1 s t}^{\prime \prime}\left(t, t_{i}\right)\right|+L_{3}\|x-y\|_{P C^{1}} \sum_{i=1}^{n}\left|G_{1 t}^{\prime}\left(t, t_{i}\right)\right| \\
\leq & \frac{2 L_{1}\|x-y\|_{P C^{1}}}{\Gamma(q)} \gamma+\frac{2 L_{1}\|x-y\|_{P C^{1}}}{\Gamma(q+1)} \gamma(n+1) c_{3}+\frac{2 L_{1}\|x-y\|_{P C^{1}}}{\Gamma(q)} \gamma n c_{2} \\
& +n c_{3} L_{2}\|x-y\|_{P C^{1}}+n c_{2} L_{3}\|x-y\|_{P C^{1}} \\
= & \left.\frac{2 L_{1}\left(1+n c_{2}\right)}{\Gamma(q)} \gamma+\frac{2 L_{1}(n+1) c_{3}}{\Gamma(q+1)} \gamma+n\left(c_{3} L_{2}+c_{2} L_{3}\right)\right]\|x-y\|_{P C^{1}} . \\
&
\end{aligned}
$$


Consequently, we have $\|T x-T y\|_{P C^{1}} \leq \Lambda\|x-y\|_{P C^{1}}$, where $\Lambda$ is defined by (4.14). As $\Lambda<1$, therefore $T$ is a contraction. Thus, the conclusion of the theorem follows by the contraction mapping principle. The proof is complete.

\section{Examples}

To illustrate how our main results can be used in practice, we present two examples.

Example 5.1 Let $q=\frac{3}{2}, n=1$. We consider the following boundary value problem:

$$
\left\{\begin{array}{l}
{ }^{c} \mathbf{D}_{0+}^{\frac{3}{2}} x(t)=d_{0} t^{\frac{1}{3}}\left[d_{1} \sqrt[5]{d_{2} t-x+x^{\prime}}-\frac{1}{20} x^{\prime}-d_{3} \ln \left(1+x^{2}\right)\right], \quad t \in J, t \neq \frac{1}{2}, \\
\left.\Delta x\right|_{t_{1}=\frac{1}{2}}=\frac{1}{10} x\left(\frac{1}{2}\right),\left.\quad \Delta x^{\prime}\right|_{t_{1}=\frac{1}{2}}=\frac{1}{6} x\left(\frac{1}{2}\right), \\
x(0)=x(1)=0,
\end{array}\right.
$$

where $d_{0}, d_{1}, d_{2}$ and $d_{3}$ are positive real numbers.

Conclusion Problem (5.1) has at least one solution in $P C^{1}[J, R] \cap C^{2}\left[J^{\prime}, R\right]$, where $J^{\prime}=$ $\left[0, \frac{1}{2}\right) \cup\left(\frac{1}{2}, 1\right]$.

Proof It follows from (5.1) that

$$
\begin{aligned}
& \omega(t)=d t^{\frac{1}{3}}, \\
& f\left(t, x, x^{\prime}\right)=d_{1} \sqrt[5]{d_{2} t-x+x^{\prime}}-\frac{1}{20} x^{\prime}-d_{3} \ln \left(1+x^{2}\right), \\
& t_{1}=\frac{1}{2}, \quad \mathcal{I}_{1}(x)=\frac{1}{10} x, \quad \mathcal{J}_{1}(x)=\frac{1}{6} x, \\
& \alpha_{1}=\alpha_{2}=1, \quad \beta_{1}=\beta_{2}=0 .
\end{aligned}
$$

From the definition of $\omega, f, \mathcal{I}_{1}$ and $\mathcal{J}_{1}$, it is easy to see that $\left(\mathrm{H}_{1}\right)-\left(\mathrm{H}_{3}\right)$ hold.

On the other hand, it follows from (5.2) and (5.3) that

$$
|f(t, x, y)| \leq d_{1} \sqrt[5]{d_{2}+|x|+|y|}+\frac{1}{20}|y|+d_{3} \ln \left(1+|x|^{2}\right)
$$

and

$$
\left|\mathcal{I}_{1}(x)\right|=\frac{1}{10}|x|, \quad\left|\mathcal{J}_{1}(x)\right|=\frac{1}{6} x, \quad \forall t \in J, x, y \in R .
$$

So $\xi \leq \frac{1}{20}, \xi_{1} \leq \frac{1}{10}, \xi_{2} \leq \frac{1}{6}$. Noticing that $\alpha_{1}=\alpha_{2}=1, \beta_{1}=\beta_{2}=0$, we have $\eta=1$ and

$$
\begin{aligned}
& G_{1}(t, s)=-\left\{\begin{array}{ll}
s(1-t), & 0 \leq s \leq t \leq 1, \\
t(1-s), & 0 \leq t \leq s \leq 1,
\end{array} \quad G_{1 t}^{\prime}(t, s)= \begin{cases}s, & 0 \leq s \leq t \leq 1, \\
s-1, & 0 \leq t \leq s \leq 1,\end{cases} \right. \\
& G_{1 s}^{\prime}(t, s)=\left\{\begin{array}{ll}
t-1, & 0 \leq s \leq t \leq 1, \\
t, & 0 \leq t \leq s \leq 1,
\end{array} \quad G_{1 s t}^{\prime}(t, s)=1 .\right.
\end{aligned}
$$

Therefore, $c_{1}=\frac{1}{4}, c_{2}=c_{3}=1$, and therefore (4.1) is satisfied because

$$
\delta_{1} \leq \frac{9}{20 \sqrt{\pi}}+\frac{11}{240}, \quad \delta_{2} \leq \frac{13}{30 \sqrt{\pi}}+\frac{4}{15}, \quad \delta \leq \frac{13}{30 \sqrt{\pi}}+\frac{4}{15}<1 .
$$

Thus, our conclusion follows from Theorem 4.1. 
Example 5.2 Let $q=\frac{5}{4}, n=1$. We consider the following boundary value problem:

$$
\left\{\begin{array}{l}
{ }^{c} \mathbf{D}_{0+x}^{\frac{5}{4}} x(t)=(\sin t+1) \frac{\left(d_{4} e^{t}+d_{5}\right)\left(d_{6}+t^{2} \sin ^{2} x\right)}{d_{6}+t^{2}+x^{2}+y^{2}}, \quad t \in J, t \neq \frac{1}{3}, \\
\left.\Delta x\right|_{t_{1}=\frac{1}{3}}=d_{7} e^{-x^{2}\left(\frac{1}{3}\right)}+d_{8} \sin ^{2} x\left(\frac{1}{3}\right), \\
\left.\Delta x^{\prime}\right|_{t_{1}=\frac{1}{3}}=\frac{d_{9}+d_{10} x^{2}\left(\frac{1}{3}\right)}{1+x^{2}\left(\frac{1}{3}\right)} \\
x(0)=x(1)=0,
\end{array}\right.
$$

where $d_{4}, d_{5}, d_{6}, d_{7}, d_{8}, d_{9}$ and $d_{10}$ are positive real numbers with $d_{10}>d_{9}$.

Conclusion Problem (5.4) has at least one solution in $P C^{1}[J, R] \cap C^{2}\left[J^{\prime}, R\right]$, where $J^{\prime}=$ $\left[0, \frac{1}{3}\right) \cup\left(\frac{1}{3}, 1\right]$.

Proof It follows from (5.4) that

$$
\begin{aligned}
& \omega(t)=\sin t+1, \\
& f(t, x, y)=\left(d_{4} e^{t}+d_{5}\right) \frac{d_{6}+t x^{2} \sin ^{2} x}{d_{6}+t^{2}+x^{2}+y^{2}}, \\
& t_{1}=\frac{1}{3}, \quad \mathcal{I}_{1}(x)=d_{7} e^{-x^{2}}+d_{8} \sin ^{2} x, \quad \mathcal{J}_{1}(x)=\frac{d_{9}+d_{10} x^{2}}{1+x^{2}}, \\
& \alpha_{1}=\alpha_{2}=1, \quad \beta_{1}=\beta_{2}=0 .
\end{aligned}
$$

From the definition of $\omega, f, \mathcal{I}_{1}$ and $\mathcal{J}_{1}$, we can obtain that $\left(\mathrm{H}_{1}\right)-\left(\mathrm{H}_{3}\right)$ hold.

On the other hand, from (5.5) and (5.6) we have

$$
|f(t, x, y)| \leq d_{4} e^{t}+d_{5}:=v_{1}(t)
$$

and

$$
\left|\mathcal{I}_{1}(x)\right| \leq \frac{d_{7}}{e}+d_{8}, \quad\left|\mathcal{J}_{1}(x)\right| \leq d_{10}, \quad \forall t \in J, x, y \in R
$$

Therefore, the conditions $\left(\mathrm{H}_{4}\right)$ and $\left(\mathrm{H}_{5}\right)$ of Theorem 4.2 are satisfied. Thus, Theorem 4.2 gives our conclusion.

Competing interests

The authors declare that they have no competing interests.

Authors' contributions

All results belong to JZ and MF. All authors read and approved the final manuscript.

\section{Acknowledgements}

This work is sponsored by the project NSFC $(11301178,11171032)$. The authors are grateful to anonymous referees for their constructive comments and suggestions which have greatly improved this paper.

Received: 6 January 2014 Accepted: 5 March 2014 Published: 25 Mar 2014

\section{References}

1. Miller, KS, Ross, B: An Introduction to the Fractional Calculus and Fractional Differential Equations. Wiley, New York (1993)

2. Oldham, KB, Spanier, J: The Fractional Calculus. Academic Press, New York (1974) 
3. Podlubny, I: Fractional Differential Equations. Mathematics in Sciences and Engineering, vol. 198. Academic Press, San Diego (1999)

4. Samko, SG, Kilbas, AA, Marichev, OI: Fractional Integrals and Derivatives: Theory and Applications. Gordon \& Breach, Yverdon (1993)

5. Kilbas, AA, Srivastava, HM, Trujillo, JJ: Theory and Applications of Fractional Differential Equations. North-Holland Mathematics Studies, vol. 204. Elsevier, Amsterdam (2006)

6. Lakshmikantham, V, Leela, S, Vasundhara Devi, J: Theory of Fractional Dynamic Systems. Cambridge Scientific Publishers, Cambridge (2009)

7. Graef, JR, Kong, L, Kong, Q, Wang, M: Theory of fractional functional differential equations. J. Appl. Anal. Comput. 3, 21-35 (2013)

8. Javidi, M, Nyamoradi, N: Dynamic analysis of a fractional order phytoplankton model. J. Appl. Anal. Comput. 3 343-355 (2013)

9. Lakshmikantham, V, Vatsala, AS: General uniqueness and monotone iterative technique for fractional differential equations. Appl. Math. Lett. 21, 828-834 (2008)

10. Ahmad, B, Nieto, JJ: Existence and approximation of solutions for a class of nonlinear impulsive functional differential equations with anti-periodic boundary conditions. Nonlinear Anal. 69, 3291-3298 (2008)

11. Ahmad, B, Nieto, J: Existence results for nonlinear boundary value problems of fractional integrodifferential equations with integral boundary conditions. Bound. Value Probl. 2009, Article ID 708576 (2009). doi:10.1155/2009/708576

12. Jiang, D, Yuan, C: The positive properties of Green's function for Dirichlet-type boundary value problems of nonlinear fractional differential equations and its application. Nonlinear Anal. 72, 710-719 (2010)

13. Bai, Z, Lü, H: Positive solutions of boundary value problems of nonlinear fractional differential equation. J. Math. Anal. Appl. 311, 495-505 (2005)

14. Bai, Z: On positive solutions of a nonlocal fractional boundary value problem. Nonlinear Anal. 72, 916-924 (2010)

15. Li, C, Luo, X, Zhou, Y: Existence of positive solutions of the boundary value problem for nonlinear fractional differential equations. Comput. Math. Appl. 59, 1363-1375 (2010)

16. Benchohra, M, Hamani, S, Ntouyas, SK: Boundary value problems for differential equations with fractional order and nonlocal conditions. Nonlinear Anal. 71, 2391-2396 (2009)

17. Salem, HAH: On the nonlinear Hammerstein integral equations in Banach spaces and application to the boundary value problem of fractional order. Math. Comput. Model. 48, 1178-1190 (2008)

18. Salem, HAH: On the fractional order $m$-point boundary value problem in reflexive Banach spaces and weak topologies. J. Comput. Appl. Math. 224, 565-572 (2009)

19. Kaufmann, ER, Mboumi, E: Positive solutions of a boundary value problem for a nonlinear fractional differential equation. Electron. J. Qual. Theory Differ. Equ. 2008(3), 1-11 (2008)

20. Liu, X, Jia, M: Multiple solutions for fractional differential equations with nonlinear boundary conditions. Comput. Math. Appl. 59, 2880-2886 (2010)

21. Jia, $\mathrm{M}, \mathrm{Liu}, \mathrm{X}$ : Three nonnegative solutions for fractional differential equations with integral boundary conditions. Comput. Math. Appl. 62, 1405-1412 (2011)

22. Zhang, S: Positive solutions to singular boundary value problem for nonlinear fractional differential equation. Comput. Math. Appl. 59, 1300-1309 (2010)

23. Feng, $M$, Zhang, $X, G e, W$ : New existence results for higher-order nonlinear fractional differential equation with integral boundary conditions. Bound. Value Probl. 2011, Article ID 720702 (2011). doi:10.1155/2011/720702

24. Lakshmikantham, V, Bainov, DD, Simeonov, PS: Theory of Impulsive Differential Equations. World Scientific, Singapore (1989)

25. Samoilenko, AM, Perestyuk, NA: Impulsive Differential Equations. World Scientific, Singapore (1995)

26. Benchohra, M, Henderson, J, Ntouyas, SK: Impulsive Differential Equations and Inclusions. Hindawi, New York (2006)

27. Lin, X, Jiang, D: Multiple positive solutions of Dirichlet boundary value problems for second order impulsive differential equations. J. Math. Anal. Appl. 321, 501-514 (2006)

28. Agarwal, RP, O'Regan, D: Multiple nonnegative solutions for second order impulsive differential equations. Appl. Math. Comput. 114, 51-59 (2000)

29. Nieto, JJ, Lópe, RR: Boundary value problems for a class of impulsive functional equations. Comput. Math. Appl. 55 2715-2731 (2008)

30. Nieto, JJ, O'Regan, D: Variational approach to impulsive differential equations. Nonlinear Anal., Real World Appl. 10, 680-690 (2009)

31. Li, J, Nieto, JJ: Existence of positive solutions for multipoint boundary value problem on the half-line with impulses. Bound. Value Probl. 2009, Article ID 834158 (2009). doi:10.1155/2009/834158

32. Shen, J, Wang, W: Impulsive boundary value problems with nonlinear boundary conditions. Nonlinear Anal. 69 4055-4062 (2008)

33. Liu, Y, O'Regan, D: Multiplicity results using bifurcation techniques for a class of boundary value problems of impulsive differential equations. Commun. Nonlinear Sci. Numer. Simul. 16, 1769-1775 (2011)

34. Guo, D: Multiple positive solutions of impulsive nonlinear Fredholm integral equations and applications. J. Math. Anal. Appl. 173, 318-324 (1993)

35. Liu, B, Yu, J: Existence of solution of $m$-point boundary value problems of second-order differential systems with impulses. Appl. Math. Comput. 125, 155-175 (2002)

36. Feng, $\mathrm{M}, \mathrm{Xie}, \mathrm{D}$ : Multiple positive solutions of multi-point boundary value problem for second-order impulsive differential equations. J. Comput. Appl. Math. 223, 438-448 (2009)

37. Agarwal, RP, Benchohra, M, Slimani, BA: Existence results for differential equations with fractional order and impulses. Mem. Differ. Equ. Math. Phys. 44, 1-21 (2008)

38. Ahmad, B, Sivasundaram, S: Existence results for nonlinear impulsive hybrid boundary value problems involving fractional differential equations. Nonlinear Anal. Hybrid Syst. 3, 251-258 (2009)

39. Ahmad, B, Sivasundaram, S: Existence of solutions for impulsive integral boundary value problems of fractional order. Nonlinear Anal. Hybrid Syst. 4, 134-141 (2010) 
40. Mophou, GM: Existence and uniqueness of mild solutions to impulsive fractional differential equations. Nonlinear Anal. 72, 1604-1615 (2010)

41. Chang, YK, Nieto, JJ: Existence of solutions for impulsive neutral integrodifferential inclusions with nonlocal initial conditions via fractional operators. Numer. Funct. Anal. Optim. 30, 227-244 (2009)

42. Abbas, S, Benchohra, M: Upper and lower solutions method for impulsive partial hyperbolic differential equations with fractional order. Nonlinear Anal. Hybrid Syst. 4, 406-413 (2010)

43. Tian, Y, Bai, Z: Existence results for the three-point impulsive boundary value problem involving fractional differential equations. Comput. Math. Appl. 59, 2601-2609 (2010)

44. Ahmad, B, Wang, G: A study of an impulsive four-point nonlocal boundary value problem of nonlinear fractional differential equations. Comput. Math. Appl. 62, 1341-1349 (2011)

45. Wang, G, Ahmad, B, Zhang, L: Some existence results for impulsive nonlinear fractional differential equations with mixed boundary conditions. Comput. Math. Appl. 62, 1389-1397 (2011)

46. Yang, L, Chen, $\mathrm{H}$ : Nonlocal boundary value problem for impulsive differential equations of fractional order. Adv. Differ. Equ. 2011, Article ID 404917 (2011). doi:10.1155/2011/404917

47. Zeidler, E: Nonlinear Functional Analysis and Its Applications - I: Fixed-Point Theorems. Springer, New York (1986)

48. Guo, D: Nonlinear Functional Analysis. Shandong Science and Technology Press, Jinan (1985) (in Chinese)

10.1186/1687-2770-2014-69

Cite this article as: Zhou and Feng: Green's function for Sturm-Liouville-type boundary value problems of fractional order impulsive differential equations and its application. Boundary Value Problems 2014, 2014:69

\section{Submit your manuscript to a SpringerOpen ${ }^{\circ}$ journal and benefit from:}

- Convenient online submission

- Rigorous peer review

- Immediate publication on acceptance

- Open access: articles freely available online

- High visibility within the field

- Retaining the copyright to your article 